\title{
Genetic screening for mutations in known long QT syndrome causing genes in patiens with known or suspected long QT syndrome with high throughput sequencing
}

Lidia Hategan ${ }^{1 *}$, István Nagy², Zoltán Hegedus³ ${ }^{3}$ Beáta Csányi', László Sághy', Miklós Csanády ${ }^{1}$, Tamás Forster ${ }^{1}$, Róbert Sepp ${ }^{1}$

${ }^{1} 2^{\text {nd }}$ Department of Internal Medicine and Cardiology Center, University of Szeged, Szeged, Hungary ${ }^{2}$ Sequencing Laboratory, Biological Research Centre, Szeged, Hungary

${ }^{3}$ Bioinformatics Laboratory, Biological Research Centre, Szeged, Hungary

The long QT syndrome (LQTS) is a disease of the cardiac ion channels with a heterogeneous genetic background. Although case reports describing mutations in known LQTS genes in Hungarian LQTS patients are available, the distribution of the disease causing genes in a larger cohort of Hungarian LQTS patients is still unknown. Our goal was to screen all LQTS causing disease genes in a cohort of Hungarian patients with definite or suspected LQTS for presence of mutations.

Thirty-six patients (12 males, 24 females, median age $40 \pm 15$ years) were screened. The patients had either a definite diagnosis of LQTS, based on prolonged QTC interval on their resting ECG $(n=23)$, developed QTc prolongation on pharmacological provocation $(n=5)$, or were survivors of sudden cardiac death in whom the suspicion of LQTS had been raised $(n=8)$.

\section{Received: $28^{\text {th }}$ Apr 2014}

*Address for correspondence: Second Department of Medicine and Cardiology Centre, University of Szeged, Korányi fasor 6, H-6720 Szeged, Hungary

Phone: $+3670-2264698$

E-mail: lidiahategan@yahoo.com
All of the 13 genes, known to cause LQTS, were sequenced. A putative disease causing mutation was defined as a nucleotide variant which was either novel, or had a minor allele frequency less than $0.5 \%$ according to the published databases. Among the 36 patients, a putative disease causing mutation was identified in 19 patients (53\%). Twentyfour mutations were found in 19 patients: 14 patients had single mutations and 5 patients had compound mutations in two different genes. The three major LQTS genes made up the majority of the identified mutations as KCNQ1 (LQT1), KCNH2 (LQT2), and SCN5A (LQT3) mutations were identified in $17 \% ; 21 \%$ and $8 \%$ of the cases, respectively. The distribution for mutations in rare LQTS genes were as follows: ANK2 (LQT4): 13\%; KCNE1 (LQT5): 4\%; KCNE2 (LQT6): 4\%; KCNJ2 (LQT7): 13\%; CACNA1C (LQT8): 4\%; AKAP9 (LQT11): 13\%; STNA1 (LQT12): 4\%.

The results of our large-scale genetic screening provide the first available data for disease gene distribution in Hungarian patients with LQTS. It also highlights the genetic heterogeneity of LQTS and demonstrates that gene-negative patients are not infrequent.

KEYWORDS: long QT syndrome, ion channel, mutation. CITATION: Cardiol Croat. 2014;9(5-6):206.

\section{Literature}

1. Refsgaard L, Holst AG, Sadjadieh G, Haunsø S, Nielsen JB, Olesen MS. High prevalence of genetic variants previously associated with LQT syndrome in new exome data. Eur J Hum Genet. 2012;20(8):905-8. 